\title{
FRECUENCIA Y SUSCEPTIBILIDAD ANTIBIÓTICA DEL Staphylococcus aureus PROVENIENTE DE HISOPADOS NASALES EN UNA POBLACIÓN URBANO MARGINAL DE LIMA, PERÚ
}

\author{
Edgar Carmona ${ }^{1, a}$, Seyzo Sandoval ${ }^{1, a}$, Coralith García $a^{1,2, b}$
}

\begin{abstract}
RESUMEN
Objetivos. Determinar la frecuencia y los factores asociados de la colonización nasal por Staphylococcus aureus, así como el patrón de susceptibilidad antibiótica en una población urbano marginal de Lima, Perú. Materiales y métodos. Estudio transversal en personas de cualquier edad provenientes de un distrito urbano marginal de la zona norte de la ciudad de Lima. El muestreo fue por conveniencia. A cada persona se le realizó una encuesta y se le tomó una muestra de hisopado de las narinas, las que fueron procesadas para la identificación de Staphylococcus aureus y determinación del patrón de susceptibilidad mediante el método de difusión de disco de Kirby Bauer. Resultados. De los 452 participantes reclutados, entre 1 y 84 años de edad, la frecuencia de colonización nasal de Staphylococcus aureus fue de 24,6\%, de los cuales solo el $0,9 \%$ fue resistente a meticilina. El único factor asociado a la colonización nasal fue la edad menor o igual a 11 años (OR: 3,80; IC95\%: 1,42 - 10,16). La mayoría de cepas fue resistente a la penicilina (96,4\%) pero también se presentó a la eritromicina $(10,9 \%)$, clindamicina $(7,3 \%)$ y gentamicina $(4,5 \%)$ en menor proporción. Conclusiones. La frecuencia de colonización nasal por Staphylococcus aureus fue similar a otros estudios a nivel mundial, con predominio de cepas meticilino sensibles.
\end{abstract}

Palabras clave: Portador sano; Staphylococcus aureus; Staphylococcus aureus resistente a meticilina; Infecciones estafilocócicas; Resistencia a antibióticos; Técnicas de laboratorio clínico (fuente: DeCS BIREME).

\section{THE FREQUENCY AND ANTIBIOTIC SUSCEPTIBILITY OF Staphylococcus aureus FROM NASAL SWABS IN AN SUBURBAN MARGINAL POPULATION IN LIMA, PERU}

\begin{abstract}
Objectives. To determine the frequency and associated factors of nasal colonization by Staphylococcus aureus and its antibiotic susceptibility pattern in a marginal suburban population Lima, Peru. Materials and methods. A cross-sectional study was conducted among population of all ages from a marginal suburban district in northern Lima. The study used a convenience sample. Each person was surveyed and provided a swab sample of the nostrils. The swab samples were analyzed to identify Staphylococcus aureus, determining the susceptibility pattern by the Kirby Bauer disk diffusion method. Results. From the 452 participants recruited, ages between 1 and 84 years, the frequency of Staphylococcus aureus nasal colonization was $24.6 \%$, with $0.9 \%$ of them resistant to methicillin. The only factor associated with nasal colonization was age equal or less than 11 years (OR: $3.80,95 \% \mathrm{Cl} 1.42$ to 10.16). Most strains were resistant to penicillin (96.4\%) but also to erythromycin $(10.9 \%)$, clindamycin $(7.3 \%)$ and gentamicin $(4.5 \%)$. Conclusions. The frequency of Staphylococcus aureus nasal colonization was similar to other studies worldwide, with predominance of methicillin-sensitive strains.
\end{abstract}

Key words: Carrier state; Staphylococcus aureus; Methicillin-resistant Staphylococcus aureus; Staphylococcal infections; Drug resistance, microbial; Clinical laboratory techniques (source: MeSH NLM).

\section{INTRODUCCIÓN}

El género Staphylococcus fue identificado por primera vez en 1882, y es el $S$. aureus la especie asociada con un mayor número de cuadros clínicos en humanos. La mayoría de las infecciones son leves, como la forunculosis, el impétigo y la foliculitis; pero puede producir cuadros graves que ponen en riesgo la vida de los pacientes, como la neumonía, la osteomielitis, las bacteriemias y la endocarditis. Si bien el S. aureus se ha relacionado con infecciones en pacientes hospitalizados en unidades de cuidados intensivos,

Facultad de Medicina Alberto Hurtado, Universidad Peruana Cayetano Heredia. Lima, Perú.

Instituto de Medicina Tropical Alexander von Humboldt, Universidad Peruana Cayetano Heredia. Lima, Perú.

Médico cirujano; ${ }^{\mathrm{b}}$ médico infectólogo

* Este estudio fue presentado como tesis para obtener el título profesional de médico cirujano en la Facultad de Medicina Alberto Hurtado de la Universidad Peruana Cayetano Heredia en 2012. 
unidades de quemados y en neutropénicos, no se debe confinar al $S$. aureus a ambientes hospitalarios, ya que el principal reservorio se encuentra en la colonización de la mucosa nasal de las personas y animales de la comunidad ${ }^{(1-3)}$.

A partir de 1990, reportes de infecciones ocasionadas por Staphylococcus aureus meticilino-resistente (SAMR) en personas sin contacto previo con algún establecimiento de salud, marcaron el surgimiento de una nueva cepa de $S$. aureus, denominada Staphylococcus aureus meticilino-resistente adquirido en comunidad $(\mathrm{SAMR}-\mathrm{AC})^{(3-6)}$.

En un inicio, los reportes de infecciones por SAMRAC involucraban infecciones relativamente leves, localizadas en piel y tejidos blandos (como abscesos, foliculitis, celulitis e impétigo); sin embargo, casos de infecciones graves de partes blandas (fasceitis necrotizante), asociados a bacteriemia y neumonía, fueron posteriormente descritos y han sido responsables del aumento en la mortalidad ${ }^{(3-6)}$.

Se han reportado casos en EE. UU., Australia, Suiza, Francia, Reino Unido, Nueva Zelanda, Finlandia, Canadá y Samoa ${ }^{(5,6)}$. En Sudamérica se ha descrito en Uruguay, Argentina, Chile, Ecuador, Colombia y Venezuela ${ }^{(7-12)}$. Es por este motivo que las infecciones por SAMR-AC, actualmente son consideradas una enfermedad emergente, que no solo involucra a países desarrollados, sino también a países en desarrollo.

A nivel mundial se estima que dos billones de personas están presentan esta bacteria, de ellas $1 \%$ es por SAMR, al ser la mucosa nasal el lugar más frecuente de colonización. Estos hechos han conllevado a una serie de investigaciones sobre la prevalencia de portadores, la relación del riesgo de infección con el estado portador y a plantear la posibilidad de eliminar este estado como medida de prevención para infecciones ${ }^{(3,5)}$. Debido a este panorama, en muchos lugares del mundo se incluye a la vancomicina como parte del tratamiento empírico de las infecciones de piel y partes blandas adquiridas en la comunidad ${ }^{(13)}$.

En Perú se han reportado los primeros casos de infecciones de $S$. aureus resistente a meticilina de origen comunitario (14), por lo que estas cepas podrían estar circulando en la comunidad a través de la colonización nasal. En vista de los reportes sobre infecciones graves de SAMR-AC en países vecinos, se vuelve urgente vigilar los reservorios de $S$. aureus. La presente investigación tiene como objetivo determinar la frecuencia de colonización nasal de S. aureus en la comunidad, investigar los factores asociados, y determinar el patrón de susceptibilidad antimicrobiana.

\section{MATERIALES Y MÉTODOS}

Se realizó un estudio transversal sobre colonización nasal por $S$. aureus en un distrito urbano marginal de la zona norte de la ciudad de Lima, entre diciembre de 2010 y junio de 2011. Se incluyó a personas de todas las edades y se excluyó a quienes habían consumido antibióticos en los últimos siete días y que tuvieran sospecha clínica de infección activa por $S$. aureus durante el período de estudio (neumonía, infección de piel o partes blandas, bacteriemia, endocarditis).

El tamaño de la muestra se calculó en 323 individuos, luego de tomar en consideración un porcentaje de portadores del $30 \%$. El reclutamiento fue por conveniencia y se realizó en cinco asentamientos humanos, dos iglesias y un instituto de educación superior.

El presente estudio contó con aprobación del Comité Institucional de Ética de la Universidad Peruana Cayetano Heredia.

\section{PROCEDIMIENTOS}

Se realizó una charla informativa sobre los alcances de la investigación en cada lugar de reclutamiento de pacientes. Luego se invitó a participar en forma voluntaria a los asistentes, y se obtuvo consentimiento informado (y asentimiento informado cuando correspondía). Se realizó una encuesta a cada participante, la cual examinaba características sociodemográficas así como antecedentes patológicos, estilos de vida y factores de riesgo para colonización nasal (15-21).

Una vez completada la encuesta por los investigadores se procedió a la toma de la muestra, la cual consistió en un hisopado de ambas fosas nasales. Cada hisopo fue introducido en un caldo de enriquecimiento Todd Hewitt y llevado al laboratorio.

\section{DEFINICIÓN DE VARIABLES}

Para la encuesta se consideraron las siguientes definiciones: crianza de animales (presencia de un animal doméstico que reside en la casa); práctica de deporte (actividades recreativas de tipo colectivas por lo menos una vez por semana); hacinamiento (hogar con más de diez personas o más de dos personas/ habitación); ingreso mensual familiar bajo (ingreso de 1179 nuevos soles para una familia de cuatro personas según la Encuesta Nacional de Hogares- 
ENAHO 2006); no fumador (persona que nunca ha fumado o ha consumido menos de 20 paquetes en su vida); fumador actual (persona que ha iniciado el hábito de fumar hace un mes como mínimo y continúa fumando), y exfumador (persona que ha fumado más de 20 paquetes en su vida pero que actualmente no sigue fumando).

\section{ANÁLISIS MICROBIOLÓGICO}

Las muestras fueron transportadas al Instituto de Medicina Tropical Alexander von Humboldt, donde se incubaron por 24 horas a $35-37^{\circ} \mathrm{C}$. Posteriormente, se realizó la inoculación por 'agotamiento' en placas de agar manitol salado y fueron incubadas por 24 a 48 horas a $35-37^{\circ} \mathrm{C}$. Las placas fueron leídas para encontrar colonias sugestivas de $S$. aureus (colonias que fermentaron manitol, virando el color del agar al amarillo). Seguidamente, estas colonias fueron nuevamente sembradas en agar manitol salado e incubadas por 24 horas a $35-37^{\circ} \mathrm{C}$ para obtener un aislamiento puro. Se realizaron las pruebas de tinción Gram, coagulasa, catalasa y DNAsa para tipificar el aislamiento como $S$. aureus.

Luego se resembraron las colonias en agar tripticasa soya (TSA) y se incubaron por 24 horas a $35-37^{\circ} \mathrm{C}$. El patrón de susceptibilidad se determinó con el método de Kirby Bauer empleando trece antibióticos: cefoxitina, oxacilina, cloranfenicol, ciprofloxacino, gentamicina, trimetoprim-sulfametoxazol, teicoplanina, penicilina, vancomicina, rifampicina, linezolid, eritromicina y clindamicina. También se realizó la prueba para resistencia inducible a clindamicina con la prueba de difusión doble de disco (D-test). Se empleó la metodología y los puntos de corte propuestos por el CLSI (Clinical and Laboratory Standard Institute 2010) ${ }^{(22) .}$

\section{ANÁLISIS ESTADÍSTICO}

Inicialmente, se estudió la distribución de cada una de las variables; en el caso de las variables categóricas se determinó el número de datos y la distribución de frecuencias según categorías, mientras que en el caso de las variables continuas se determinó su aproximación a la distribución normal.

El análisis de la asociación entre la variable respuesta y las variables de exposición se realizó a través de un análisis bivariado (prueba de chi cuadrado) para luego concluir en un análisis multivariado (regresión logística). Se consideró significancia estadística si el valor de $p$ fue menor a 0,05. Para el análisis de los datos se utilizó el programa estadístico STATA versión 12.0 .
Tabla 1. Características sociodemográficas de la población.

\begin{tabular}{|c|c|}
\hline Características & n (\%) \\
\hline \multicolumn{2}{|l|}{ Edad } \\
\hline Niños (1-11 años) & $89(19,7)$ \\
\hline Adolescentes (12-17 años) & $40(8,8)$ \\
\hline Jóvenes (18 a 29 años) & $92(20,4)$ \\
\hline Adultos (30 a 59 años) & $179(39,6)$ \\
\hline Adultos mayores (60 a 84 años) & $52(11,5)$ \\
\hline \multicolumn{2}{|l|}{ Ocupación } \\
\hline Estudiante & $157(34,7)$ \\
\hline Ama de casa & $142(31,4)$ \\
\hline Otras & $153(33,9)$ \\
\hline \multicolumn{2}{|l|}{ Ingreso mensual } \\
\hline Bajo (<1179 nuevos soles) & $373(82,5)$ \\
\hline \multicolumn{2}{|l|}{ Crianza de animales } \\
\hline Posee al menos un animal & $308(68,1)$ \\
\hline \multicolumn{2}{|l|}{ Práctica de deporte } \\
\hline Al menos una vez por semana & $198(43,8)$ \\
\hline \multicolumn{2}{|l|}{ Hospitalizaciones previas } \\
\hline Alguna vez hospitalizado & $105(23,2)$ \\
\hline \multicolumn{2}{|l|}{ Intervenciones quirúrgicas } \\
\hline Alguna vez operado & $92(20,4)$ \\
\hline \multicolumn{2}{|l|}{ Consulta externa } \\
\hline Acudió el último año & $186(41,1)$ \\
\hline Total & $452(100)$ \\
\hline
\end{tabular}

\section{RESULTADOS}

Se reclutaron 452 participantes durante el periodo de estudio, de los cuales 345 provinieron de asentamientos humanos $(76,3 \%), 72$ de iglesias $(15,9 \%)$ y 35 de un instituto de educación superior $(7,7 \%)$. La mayoría de participantes fue de sexo femenino $(56,2 \%)$, con edades que oscilaron entre 1 y 84 años (edad promedio: $32,1 \pm$ 20,3 años). El promedio de habitantes por casa fue de 5 \pm 2 y el número de personas por habitación $2 \pm 1$. Las características de la población se ven en la Tabla 1.

\section{FACTORES ASOCIADOS A LA COLONIZACIÓN NASAL}

La frecuencia de colonización nasal por $S$. aureus fue de 24,6\% (IC95\%: 20,6 - 28,6). De los 111 aislamientos de $S$. aureus, solo uno fue resistente a meticilina $(0,9 \%)$. Según el análisis bivariado, de todas las variables incluidas en la encuesta, se obtuvieron solo tres con significancia estadística (Tabla 2). Se realizó regresión logística univariada, donde se obtuvo que la edad, específicamente la de los niños (OR: 4,17; IC95\%: $1,69-10,28 ; p=0,002)$; el hacinamiento mayor a diez personas por vivienda (OR: 3,32; IC95\%: 1,45 - 7,63; $\mathrm{p}=0,005)$ y el practicar deporte (OR: 1,73: IC95\%: 1,12 - 2,66; $p=0,013$ ) presentaron intervalos de confianza por encima de 1 (Tabla 3). 
Tabla 2. Análisis bivariado: factores asociados a colonización nasal por Staphylococcus aureus.

\begin{tabular}{|c|c|c|c|c|}
\hline Factor & Total (\%) & $\begin{array}{c}\text { Positivo } \\
\text { n (\%) }\end{array}$ & $\begin{array}{c}\text { Negativo } \\
\text { n (\%) }\end{array}$ & $p$ \\
\hline Edad & & & & 0,001 \\
\hline Niños (1-11 años) & $89(19,7)$ & $35(31,5)$ & $54(15,8)$ & \\
\hline Adolescentes (12-17 años) & $40(8,8)$ & $12(10,8)$ & $28(8,2)$ & \\
\hline Jóvenes (18 a 29 años) & $92(20,4)$ & $24(21,6)$ & $68(19,9)$ & \\
\hline Adultos (30 a 59 años) & $179(39,6)$ & $33(29,7)$ & $146(42,8)$ & \\
\hline Adultos mayores (60 a 84 años) & $52(11,2)$ & $7(6,3)$ & $45(13,2)$ & \\
\hline Sexo & & & & 0,123 \\
\hline Masculino & $198(43,8)$ & $56(50,5)$ & $142(41,6)$ & \\
\hline Femenino & $254(56,2)$ & $55(49,5)$ & $199(58,4)$ & \\
\hline \multicolumn{5}{|l|}{ Hacinamiento } \\
\hline$>10$ personas por hogar & $24(5,3)$ & $12(10,8)$ & $12(3,5)$ & 0,006 \\
\hline >2 personas por habitación & $142(31,4)$ & $40(36,0)$ & $102(29,9)$ & 0,240 \\
\hline Hábito de fumar & & & & 0,545 \\
\hline No fumador & $356(78,8)$ & $91(82,0)$ & $265(77,7)$ & \\
\hline Exfumador & $72(15,9)$ & $16(14,4)$ & $56(16,4)$ & \\
\hline Fumador actual & $24(5,3)$ & $4(3,6)$ & $20(5,9)$ & \\
\hline Ingreso mensual bajo & $373(82,5)$ & $95(85,6)$ & $278(81,5)$ & 0,389 \\
\hline Crianza de animales & $308(68,1)$ & $83(74,8)$ & $225(66,0)$ & 0,100 \\
\hline Práctica de deporte & $198(43,8)$ & $60(54,1)$ & $138(40,5)$ & 0,015 \\
\hline Hospitalizaciones previas & $105(23,2)$ & $23(20,7)$ & $82(24,0)$ & 0,519 \\
\hline Intervenciones quirúrgicas & & & & 0,138 \\
\hline Alguna vez operado & $92(20,4)$ & $17(15,3)$ & $75(22,0)$ & \\
\hline Consulta externa & & & & 0,414 \\
\hline Acudió el último año & $186(41,1)$ & $42(37,8)$ & $144(42,2)$ & \\
\hline
\end{tabular}

Según la regresión logística multivariada se observó que el único factor asociado con significancia estadística fue la edad menor o igual a 11 años (OR: 3,80; IC95\%: 1,42 - 10,16; $p=0,008$ ) (Tabla 3).

\section{PATRÓN DE SUSCEPTIBILIDAD}

El patrón de susceptibilidad para las $110(99,1 \%)$ cepas de $S$. aureus meticilino sensible (SAMS) se muestra en la Figura 1. La gran mayoría de cepas es resistente a la penicilina $(96,4 \%)$. También se observó resistencia a eritromicina $(10,9 \%)$, clindamicina $(7,3 \%)$ y gentamicina
$(4,5 \%)$. Los aislamientos resistentes a clindamicina $(\mathrm{n}=8)$ tuvieron resistencia inducible.

No se encontró resistencia a linezolid, rifampicina ni trimetoprim-sulfametoxazol. Solo se detectó una cepa de SAMR $(0,9 \%)$, sensible a linezolid, teicoplanina y cloranfenicol; pero resistente a trimetoprim-sulfametoxazol, rifampicina, ciprofloxacino, gentamicina, penicilina, eritromicina y clindamicina. El portador de esta cepa era un adulto mayor con insuficiencia cardiaca que había estado hospitalizado en el último mes.

Tabla 3. Factores asociados con la presencia de Staphylococcus aureus en hisopados nasales, Lima, Perú.

\begin{tabular}{|c|c|c|c|c|c|c|}
\hline Factor & OR crudo & IC95\% & $p$ & OR ajustado & IC95\% & $p$ \\
\hline \multicolumn{7}{|l|}{ Edad } \\
\hline Niños (1-11 años) & 4,17 & $(1,69-10,28)$ & 0,002 & 3,80 & $(1,42-10,16)$ & 0,008 \\
\hline Adolescentes (12-17 años) & 2,76 & $(0,97-7,83)$ & 0,057 & 2,64 & $(0,86-8,14)$ & 0,091 \\
\hline Jóvenes (18 a 29 años) & 2,27 & $(0,90-5,71)$ & 0,082 & 2,21 & $(0,84-5,83)$ & 0,108 \\
\hline Adultos (30 a 59 años) & 1,45 & $(0,60-3,51)$ & 0,406 & 1,46 & $(0,60-3,58)$ & 0,408 \\
\hline Adultos mayores (60 a 84 años) & 1 & - & & 1 & - & \\
\hline \multicolumn{7}{|l|}{ Hacinamiento } \\
\hline$>10$ personas por hogar & 3,32 & $(1,45-7,63)$ & 0,005 & 2,48 & $(0,89-6,94)$ & 0,084 \\
\hline \multicolumn{7}{|l|}{ Practica deporte } \\
\hline Al menos una vez por semana & 1,73 & $(1,12-2,66)$ & 0,013 & 1,09 & $(0,65-1,86)$ & 0,757 \\
\hline
\end{tabular}

IC95\%: Intervalo de confianza al 95\% 
Figura 1. Patrón de susceptibilidad antimicrobiana de los aislamientos de $S$. aureus sensibles a meticilina.

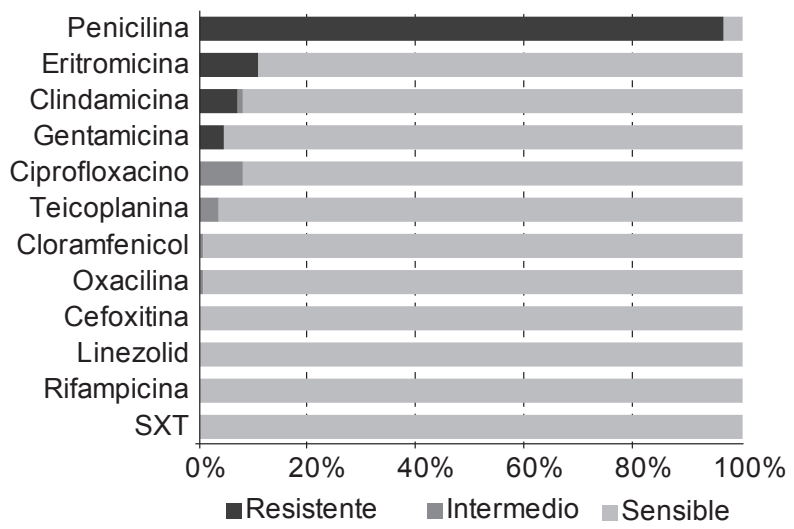

\section{DISCUSIÓN}

En el presente estudio se halló una frecuencia de $24,6 \%$ de portadores nasales de $S$. aureus; similar a los resultados obtenidos por diversos autores ${ }^{(23,24)}$. Luego del análisis estadístico, se encontró que el factor asociado a la colonización por $S$. aureus fue la edad, específicamente en el rango de 1 a 11 años.

En cuanto a la edad y el estado portador, se halló una relación inversamente proporcional, la cual es congruente con lo descrito en la literatura ${ }^{(24,25)}$. Sin embargo, existe una serie de estudios en población adulta mayor, residentes de casas de reposo, en las que la prevalencia de portadores varía entre 20 a $30 \%{ }^{(26,27)}$, por lo que la distribución de portadores en comunidad se comportaría como una distribución bimodal, no observada en el estudio. Esto se debería al número pequeño de participantes adultos mayores en la muestra y que en Perú el uso de casas de reposo no se encuentra difundido.

En cuanto a la mayor tasa de portadores en niños, esta podría deberse a que un ambiente cerrado como la comunidad escolar, un hábito de higiene inadecuado y una mayor interacción con animales facilitarían la diseminación de este organismo. No se cuenta, al momento, con más información para determinarlo con certeza. Asimismo, la condición de hacinamiento y la práctica de deporte deberían volver a investigarse en estudios futuros por su posible relevancia en estudios previos ${ }^{(28-30)}$.

Con relación a la susceptibilidad antibiótica, todas las cepas encontradas fueron SAMS a excepción de una cepa SAMR cuyo perfil de resistencia era concordante con una cepa intrahospitalaria, siendo el portador de esta un paciente con factores de riesgo para ser colonizado con cepas del ambiente hospitalario. Se encontraron ocho aislamientos resistentes a clindamicina, todos presentaron resistencia inducible a partir de una cepa resistente a eritromicina. Por esta razón, debería considerarse la implementación del D-test en los laboratorios de microbiología ya que es una técnica simple que permitiría detectar aislamientos falsamente catalogados como susceptibles, al emplear la prueba de susceptibilidad de Kirby Bauer convencional; lo cual evitaría prescribir erróneamente un antibiótico al cual la cepa es resistente.

Algunas limitaciones del presente estudio contemplan el tipo de muestreo no probabilístico que impide la extrapolación de los datos, la falta de evaluación de otros sitios de colonización de S. aureus (como piel, axila y recto) y el seguimiento a los pacientes portadores para determinar el estado persistente o transitorio.

En conclusión, la frecuencia de colonización nasal por $S$. aureus obtenida es similar a otros estudios a nivel mundial con predominio aun de cepas meticilino sensibles. Se recomienda mantener la vigilancia sobre este reservorio comunitario ya que el SAMR constituye un problema emergente a nivel mundial.

\section{AGRADECIMIENTOS}

A las Lic. Gertrudis Horna y Lizeth Astocondor por el apoyo en el análisis microbiológico de las muestras.

\section{Contribuciones de autoría}

EC y SS participaron en la concepción, diseño y ejecución del trabajo, análisis e interpretación de datos, y redacción del manuscrito. CG participó en la concepción y diseño del trabajo, interpretación de datos, redacción del manuscrito y obtención del financiamiento. Todos revisaron y aprobaron la versión final del trabajo.

\section{Fuentes de financiamiento}

Estudio subvencionado por el proyecto colaborativo del Instituto de Medicina Tropical Alexander von Humboldt - FA3 (Cooperación Belga).

\section{Conflictos de interés}

Los autores declaran no tener conflictos de interés en la publicación de este artículo.

\section{REFERENCIAS BIBLIOGRÁFICAS}

1. Wertheim H, Melles D, Vos M, Leeuwen W, Belkum A, Verbrugh $\mathbf{H}$, et al. The role of nasal carriage in Staphylococcus aureus infections. Lancet Infect Dis. 2005;5(12):751-62.

2. Williams R. Healthy carriage of Staphylococcus aureus: its prevalence and importance. Bacteriol Rev. 1963;27:56-71. 
3. Kluytmans-VandenBergh M, Kluytmans J. Communityacquired methicillin-resistant Staphylococcus aureus: current perspectives. Clin Microbiol Infect. 2006;12 Suppl 1:9-15.

4. Rozenbaum R, Sampaio M, Batista G, Garibaldi A, Terra G, Souza M, et al. The first report in Brazil of severe infection caused by community-acquired methicillinresistant Staphylococcus aureus (CA-MRSA). Braz J Med Biol Res. 2009;42(8):756-60.

5. Matouskova I, Janout V. Current knowledge of methicillinresistant Staphylococcus aureus and communityassociated methicillin-resistant Staphylococcus aureus. Biomed Pap Med Fac Univ Palacky Olomouc Czech Repub. 2008;152(2):191-202.

6. Moellering R. Current treatment options for communityacquired methicillin-resistant Staphylococcus aureus infection. Clin Infect Dis. 2008;46(7):1032-7.

7. Paganini H, Latta M, Opet B, Ezcurra G, Uranga M, Aguirre C, et al. Estudio multicéntrico sobre las infecciones pediátricas por Staphylococcus aureus meticilino-resistente provenientes de la comunidad en la Argentina. Arch Argent Pediatr. 2008;106(5):397-403.

8. Ma X, Galiana A, Pedreira W, Mowszowicz M, Christophersen I, Machiavello S, et al. Communityacquired Methicillin-resistant Staphylococcus aureus, Uruguay. Emerg Infect Dis. 2005;11(6):973-6.

9. Noriega L, González P, Hormazábal J, Pinto C, Canals M, Munita J, et al. Staphylococcus aureus comunitario resistente a cloxacilina: Comunicación de los primeros cinco casos descritos en Chile. Rev Méd Chile. 2008;136(7):885-91.

10. Gardella N, von Specht M, Cuiroloc A, Rosatoc A, Gutkind G, Molleracha M. Community-associated methicillin-resistant Staphylococcus aureus, eastern Argentina. Diagn Microbiol Infect Dis. 2008;62(3):343-7.

11. Reyes J, Rincón S, Díaz L, Panesso D, Contreras GA, Zurita J, et al. Dissemination of methicillin-resistant Staphylococcus aureus USA300 sequence type 8 lineage in Latin America. Clin Infect Dis. 2009;49(12):1861-7.

12. Ribeiro A, Dias C, Silva-Carvalho MC, Berquó L, Ferreira FA, Santos RN, et al. First report of infection with community acquired methicillin-resistant Staphylococcus aureus in South America. J Clin Microbiol. 2005;43(4):1985-8.

13. Liu C, Bayer A, Cosgrove S, Daum R, Fridkin S, Gorwitz $\mathbf{R}$, et al. Clinical practice guidelines by the infectious diseases society of america for the treatment of methicillin-resistant Staphylococcus aureus infections in adults and children: executive summary. Clin Infect Dis. 2011;52(3):285-92.

14. García C, Deplano A, Denis O, León M, Siu H, Chincha O, et al. Spread of community-associated methicillin-resistant Staphylococcus aureus to Peru. J Infect. 2011;63(6):482-3.

15. Bischoff W, Wallis M, Tucker K, Reboussin B, Sherertz R. Staphylococcus aureus nasal carriage in a student community: prevalence, clonal relationships, and risk factors. Infect Control Hosp Epidemiol. 2004;25(6):485-91.

16. Gorwitz R, Kruszon-Moran D, McAllister S, McQuillan G, McDougal L, Fosheim G, et al. Changes in the Prevalence of Nasal Colonization with Staphylococcus aureus in the United States, 2001-2004. J Infect Dis. 2008;197(9):1226-34.

17. Salgado C, Farr B, Calfee D. Community-acquired methicillin-resistant Staphylococcus aureus: a metaanalysis of prevalence and risk factors. Clin Infect Dis. 2003;36(2):131-9.
18. Seng Choi C, Suet Yin C, Abu Bakar A, Sakewi Z, NyiNaing N, Jamal F, et al. Nasal carriage of Staphylococcus aureus among healthy adults. J Microbiol Inmunol Infect. 2006;39(6):458-64.

19. Leman R, Alvarado-Ramy F, Pocock S, Barg N, Kellum M, McAllister S, et al. Nasal carriage of methicillin-resistant Staphylococcus aureus in an American Indian population. Infect Control Hosp Epidemiol. 2004;25(2):121-5.

20. Graham P, Lin S, Larson E. A U.S. Population-Based Survey of Staphylococcus aureus Colonization. Ann Intern Med. 2006;144(5):318-25.

21. Fritz S, Garbutt J, Elward A, Shannon W, Storch G. Prevalence of and risk factors for community-acquired methicillin-resistantand methicillin-sensitive Staphylococcus aureus colonization in children seen in a practice-based research network. Pediatrics. 2008;121(6):1090-8.

22. Clinical and Laboratory Standards Institute. Performance standards for antimicrobial susceptibility testing: eighteenth informational supplement. 18 ed. Wayne, PA: Clinical and Laboratory Standards Institute; 2008.

23. Skråmm I, Moen AE, Bukholm G. Nasal carriage of Staphylococcus aureus: frequency and molecular diversity in a randomly sampled Norwegian community population. APMIS. 2011;119(8):522-28.

24. Munckhof W, Nimmo G, Schooneveldt J, Schlebusch S, Stephens A, Williams G, et al. Nasal carriage of Staphylococcus aureus, including community-associated methicillin-resistant strains, in Queensland adults. Clin Microbiol Infect. 2009;15(2):149-55.

25. Halablab M, Hijazi S, Fawzi M, Araj G. Staphylococcus aureus nasal carriage rate and associated risk factors in individuals in the community. Epidemiol Infect. 2010;138(5):702-6.

26. Hsu CC. Serial survey of methicillin-resistant Staphylococcus aureus nasal carriage among residents in a nursing home. Infect Control Hosp Epidemiol. 1991;12(7):416-21.

27. Daeschlein G, Assadian $O$, Rangous I, Kramer A. Risk factors for Staphylococcus aureus nasal carriage in residents of three nursing homes in Germany. J Hosp Infect. 2006;63(2):216-20.

28. Creech C, Saye E, McKenna B, Johnson B, Jimenez N, Talbot T, et al. One-year surveillance of methicillin-resistant Staphylococcus aureus nasal colonization and skin and soft tissue infections in collegiate athletes. Arch Pediatr Adolesc Med. 2010;164(7):615-20.

29. Benjamin HJ, Nikore V, Takagishi J. Practical management: community-associated methicillin-resistant Staphylococcus aureus (CA-MRSA): the latest sports epidemic. Clin J Sport Med.2007;17(5):393-7.

30. Huijsdens $X$, van Lier A, van Kregten E, Verhoef $L$, van Santen-Verheuvel M, Spalburg E, et al. Methicillinresistant Staphylococcus aureus in Dutch soccer team. Emerg Infect Dis. 2006;12(10):1584-6.

Correspondencia: Edgar Carmona Huamán

Dirección: Los Ciruelos 151 Residencial Monterrico, Lima 12, Perú.

Teléfono: (511) 4378139 - 987846445

Correo electrónico: edgar_carmona@hotmail.com 